\title{
Data Assimilation in the Welding Process for Analysis of Weld Toe Geometry and Heat Source Model
}

\author{
Takayuki SHIRAIWA, ${ }^{1) *}$ Manabu ENOKI, ${ }^{1)}$ Sota GOTO') and Takashi HIRAIDE ${ }^{3)}$ \\ 1) Department of Materials Engineering, School of Engineering, The University of Tokyo, 7-3-1 Hongo, Bunkyo-ku, Tokyo, 113- \\ 8656 Japan. \\ 2) Steel Research Laboratory, JFE Steel Corporation, 1-1 Minamiwatarida-cho, Kawasaki-ku, Kawasaki, Kanagawa, $210-0855$ \\ Japan. \\ 3) Steel Research Laboratory, JFE Steel Corporation, 1 Kawasaki-cho, Chuo-ku, Chiba, 260-0835 Japan.
}

(Received on November 5, 2019; accepted on December 23, 2019; J-STAGE Advance published date: February 18, 2020)

\begin{abstract}
Finite element simulations are widely conducted to evaluate the heat transfer and deformation during welding. Basically these welding simulations require input variables such as shape parameters and heat source parameters, which are not directly measured by the experimental method. In this study, two methods were proposed to obtain these input parameters more efficiently: a method of automatically identifying toe radius and reinforcement angle from height profile, and a method of estimating a heat source model in welding simulation. In the first method, the toe radius and reinforcement angle were extracted from the height profile by Akaike's information criterion. The extracted results were consistent with the manual fitting results. In the second method, the optimal combination of the heat input parameters was automatically searched by Bayesian optimization. Comparing the accumulated regrets, it was found that the probability of improvement and upper confidence bound provide more efficient optimization than the other acquisition functions in the calibration of the heat input parameters. Both temperature history and shape of fusion zone and heat-affected zone calculated at the optimized condition were in good agreement with the experimental results. These results demonstrated that the two proposed methods are effective to create a numerical model for welding simulation.
\end{abstract}

KEY WORDS: welded joints; residual stresses; finite element analysis; Bayesian optimization; fatigue.

\section{Introduction}

The fatigue properties of welded joints are affected by various factors such as geometries, defects, residual stress, and microstructure, which make fatigue prediction more complicated than the base materials. The geometries can be roughly classified into global geometries such as joint type and plate thickness, and local geometries such as toe radius and reinforcement angle at the weld toe. ${ }^{1,2)}$ While the global geometries can be easily obtained, the determination of the local geometries requires precise measurement and manual fitting. These local and global geometries affect stress concentration, and the resultant fatigue crack initiation and propagation. The defects such as undercut, slag inclusion, spatter and microcracks play an important role in the crack initiation. ${ }^{3)}$ The residual stress is mainly related to fatigue crack propagation. For example, low transformation temperature (LTT) welding materials improves the crack propagation properties by reducing the tensile residual stress. ${ }^{4)}$ It is clear that the residual stress affects the crack propagation. Additionally, the welded part contains a com-

* Corresponding author: E-mail: shiraiwa@rme.mm.t.u-tokyo.ac.jp DOI: https://doi.org/10.2355/isijinternational.ISIJINT-2019-720 plicated microstructure due to the non-uniform temperature distribution and cooling rate. In the case that the welded part does not contain significant defects, the fatigue crack initiation is strongly affected by the microstructure. Also, in fatigue crack propagation, there is the effect of microstructure due to crack deflection and crack closure. ${ }^{5,6)}$ Thus, a number of factors affect the fatigue crack initiation and propagation of the welded joint in a complex manner. To establish an accurate fatigue prediction methodology for the welded joints, it is necessary to quantitatively evaluate the effect of these factors on fatigue properties.

Under these circumstances, various numerical methods have been proposed to predict the fatigue properties in the welded joints. For example, a fracture mechanics-based approach has been recently developed in IBESS project, ${ }^{7,8)}$ and the experimental validation showed that the IBESS model provided the accurate prediction of the fatigue crack propagation and fatigue life. The problem in such fracture mechanics-based methods is that they require an assumption of initial crack size to calculate stress intensity factor, and do not explicitly consider the effects of microstructure. To overcome these limitations, microstructure-based fatigue modeling has recently been developed with micromechanics, ${ }^{9,10)}$ crystal plasticity, ${ }^{11-13)}$ and data-driven approaches, ${ }^{14)}$ and 
these techniques have been applied for fatigue life prediction of welded joints. ${ }^{14,15)}$ A common problem in these numerical calculations is the preparation of the input variables such as weld toe geometries, heat source parameters in welding simulation, crystal plasticity parameters, and crack initiation criterion, which are difficult to measure directly by experiment. In order to apply the fatigue prediction methods to a wider range of materials and joint types, a method to obtain the input variables more efficiently is necessary.

Among these input variables, this study focused on the weld toe geometries and the heat source parameters of welding simulation. The weld toe geometry is represented by the toe radius and the reinforcement angle in almost cases because the fatigue strength of welded joints decreases with decreasing the toe radius and increasing the reinforcement angle. Although the height profile around the weld toe can be measured by laser displacement meter and plastic replica technique, the problem is that there are no standardized definitions or generally accepted methods for the determination of the toe radius and the reinforcement angle. ${ }^{16)}$ Currently, skilled inspectors estimate these parameters by manually fitting a circle to the measured height profile data. However, it is pointed out that the fitting results differ significantly depending on the inspectors, even if the same measurement data is used. ${ }^{17)} \mathrm{A}$ method to clearly define these parameters without the decision of a researcher or inspector is desired. The heat source parameters of the welding simulation include the shape parameters of the heat source model and the conversion efficiency from electrical power to heat input. These parameters are estimated from the prior knowledge of the researcher, or determined by trial and error to minimize the difference in temperature data between simulation and experiment. A more sophisticated method to efficiently calibrate the heat source parameters without manual trial and error is required.

Recently, Islam et al. proposed an optimization framework that minimizes welding distortion by applying genetic algorithm (GA) to finite element method (FEM). ${ }^{18)}$ However, GA tends to fall into a local solution due to the premature convergence problem. Efficient estimation of models and parameters in numerical simulation is also required in fields other than materials engineering. In geoscience, data assimilation techniques have been developed for weather forecasting, ${ }^{19)}$ earthquake analysis ${ }^{20)}$ and tsunami modeling ${ }^{21,22)}$ with the rapid improvement of computer performance. The data assimilation is defined as a method of creating or improving a model by combining numerical simulations and observation data, and more narrowly refers to the assimilation of time series data and time evolution model in meteorology and seismology. In materials engineering, data assimilation is beginning to be used to estimate parameters such as the thermal conductivity in heat transfer analysis, ${ }^{23)}$ parameters in micromechanical modeling, ${ }^{24,25)}$ and the mobility of the phase-field simulation. ${ }^{26-28)}$

The present study aims to propose automatic characterization methods of weld toe geometry and heat source model by data assimilation techniques. In the analysis of weld toe geometry, the utilization of information criterion is examined to detect the position of the weld toe. The automatically detected parameters of toe geometry are compared with the results of conventional manual fitting to validate the proposed method. In the characterization of the heat source model, numerical simulations of heat transfer, phase transformation, and elastic-plastic deformation are repeatedly conducted with the Bayesian optimization method. Furthermore, the effect of selection of the objective function on the residual stress calculation is examined in bead-on-plate welding. The analyses of the weld toe geometry and the heat source model are presented in Sections 2 and 3, respectively.

\section{Automatic Characterization of Weld Toe Geometry}

\subsection{Geometric Model}

A simple model was considered to express the geometry of weld toe as shown in Fig. 1. In the model, the height profile in the direction perpendicular to the weld line was divided into three regions named as "base," "toe," and "bead" in the figure. Assuming that the base part is a straight line parallel to the $x$ axis, the toe part is a circular arc with a radius of $r$, and the bead part is a straight line which smoothly connects with the circular arc, the height profile can be written from geometrical considerations as follows:

$$
y= \begin{cases}r+y_{0}-\frac{r^{2}+w_{2}\left(x-x_{c}\right)}{\sqrt{r^{2}-w_{2}^{2}}} & \left(x_{c}-w_{1}-w_{2} \leq x<x_{c}-w_{2}\right) \\ r+y_{0}-\sqrt{r^{2}-\left(x-x_{c}\right)^{2}} & \left(x_{c}-w_{2} \leq x<x_{c}\right) \\ y_{0} & \left(x_{c} \leq x \leq x_{c}+w_{3}\right)\end{cases}
$$

where $x_{\mathrm{c}}$ is $x$ coordinate of the transition between base and toe, $w_{1}, w_{2}, w_{3}$ are region width of bead, toe, and base, respectively. The coordinates of the transition between bead and toe is equal to $\left(x_{c}-w_{2}, r+y_{0}-\sqrt{r^{2}-w_{2}^{2}}\right)$. The parameters of $r$ and $\theta=\arctan \left(w_{2} / \sqrt{r^{2}-w_{2}^{2}}\right)$ represents the toe radius and the reinforcement angle.

\subsection{Extraction of Geometric Parameters by Akaike's Information Criterion (AIC)}

To find the best combination of $\left(x_{\mathrm{c}}, r\right)$ to fit the measurement data automatically, the Akaike's information criterion
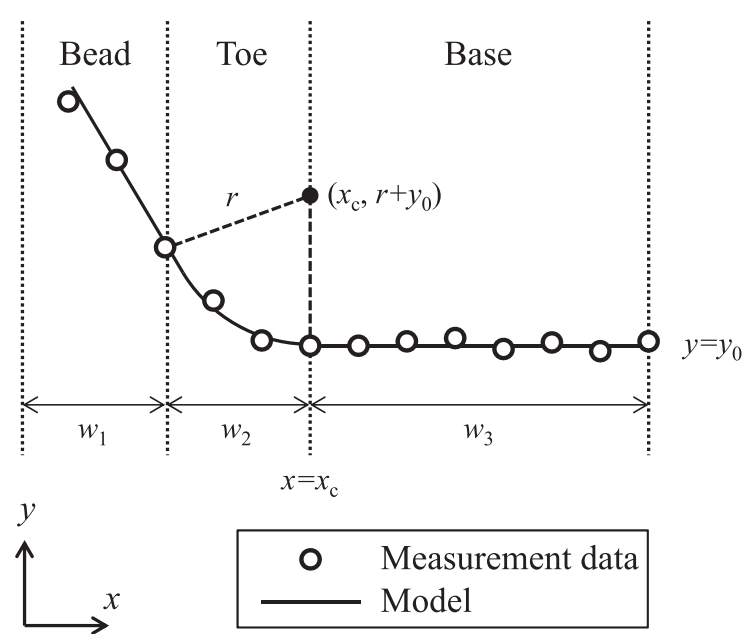

Fig. 1. Schematic of measurement data of the weld toe profile and the geometric model. 
$(\mathrm{AIC})^{29,30)}$ was used. The AIC is generally used to consider a balance between the goodness of fit and simplicity of the model. The model with the lowest AIC value is selected as the preferred model among a set of model candidates. For example, the rise time of seismic wave and acoustic emission signals can be determined at the lowest AIC value by assuming the models of noise and signal. ${ }^{31,32)}$ Under the assumption that the difference between the geometrical model of weld toe and the observed height values follows normal distributions with different parameters in the three regions (bead, toe, base), the likelihood function for the geometrical model is expressed by the following equations:

$$
L\left(x_{c}, r, w_{1}, w_{2}, w_{3}\right)=\prod_{i=1}^{3} \prod_{j=1}^{s_{i}}\left\{\frac{1}{\sqrt{2 \pi \sigma_{S D i}^{2}}} \exp \left(-\frac{y_{\mathrm{obs}, j}^{\prime}-\mu_{i}}{2 \sigma_{S D i}^{2}}\right)\right\}
$$

$$
y_{o b s, j}^{\prime}=y_{j}-y_{\mathrm{obs}, j}
$$

where $i$ is index of three regions (base, toe, and bead), $j$ is index of observed data, $s_{i}$ is number of data point in region $i$, $y_{o b s, j}^{\prime}$ is difference between observed height value and model value calculated by Eq. (1), $\mu_{i}$ and $\sigma_{\mathrm{SD}} i$ are mean value and standard deviation of $y_{o b s, j}^{\prime}$ in region $i$, respectively. The difference between the measurement value and the model value in height profile is considered to be surface roughness and measurement errors. The AIC is represented as a function of $x_{\mathrm{c}}, r, w_{1}, w_{2}$, and $w_{3}$ :

$$
\operatorname{AIC}\left(x_{c}, r, w_{1}, w_{2}, w_{3}\right)=-2 \ln L+2 k,
$$

(a) BT1

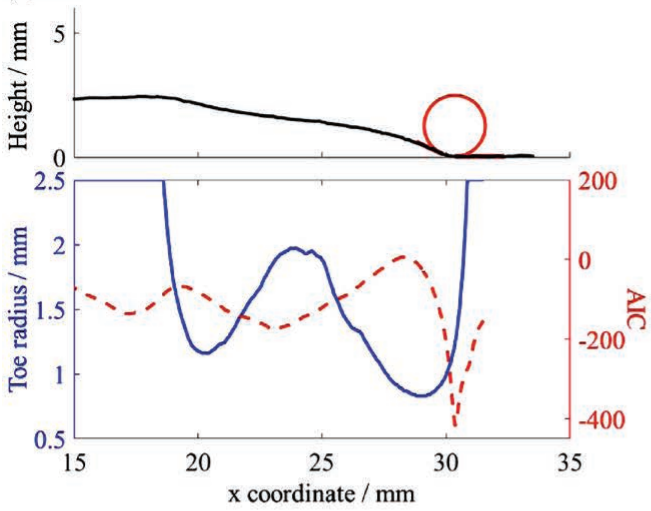

(c) CR1

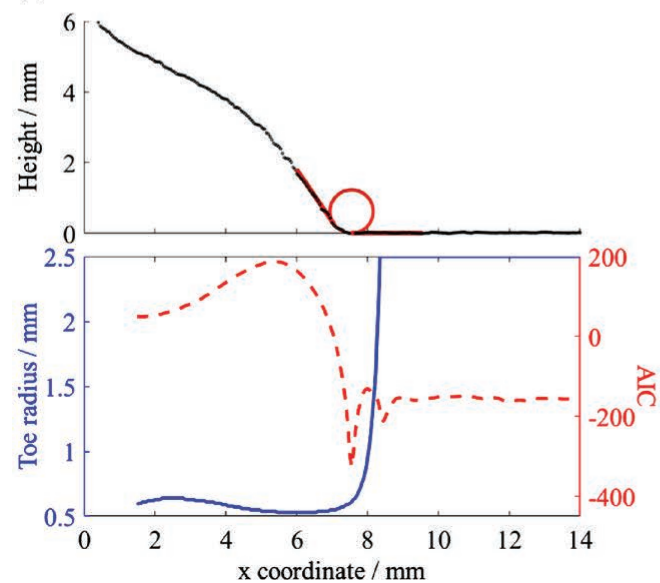

where $k$ is the number of parameters in the model. By changing the $x_{\mathrm{c}}, r$, and $w_{i}$ within a certain range and selecting a model with the minimum AIC value, the optimal toe position $\left(x_{\mathrm{c}}\right)$, toe radius $(r)$, and the reinforcement angle $\left(\theta=\arctan \left(w_{2} / \sqrt{r^{2}-w_{2}^{2}}\right)\right)$ can be obtained automatically.

\subsection{Comparison with Manual Fitting}

To validate the proposed method, two butt weld joints and two cruciform joints were prepared using low carbon steel (Fe-0.15C-0.25Si-1.0Mn) plates with a thickness of $22 \mathrm{~mm}$. The height profile around the weld toe of each joint was measured by a laser displacement meter with $0.05 \mathrm{~mm}$ step in the direction perpendicular to the weld line. Using the height profile, the toe radius and reinforcement angle were estimated by the proposed AIC method. In order to simplify the calculation, $w_{1}$ and $w_{3}$, which have relatively small effects on the fitting result, are fixed at $1.0 \mathrm{~mm}$ and 2.0 $\mathrm{mm}$, respectively. The $x_{\mathrm{c}}$ was changed within the measurement range, $r$ and $w_{2}$ were changed in the range of 0.5 to $2.5 \mathrm{~mm}$ and 0.25 to $1.25 \mathrm{~mm}$, respectively, to search for a combination of $\left(x_{\mathrm{c}}, r, w_{2}\right)$ having the lowest AIC value. The measurement data of height profile, the fitted toe radius, and AIC value for the two butt weld joints and the two cruciform joints are shown in Fig. 2. In the upper plot of each joint, the black dot shows the measurement data of the height profile, and the circle and lines represent the geometric model with the lowest AIC value. In the lower plot of each joint, the solid line and dashed line represent the fitted toe radius and

\section{(b) BT2}

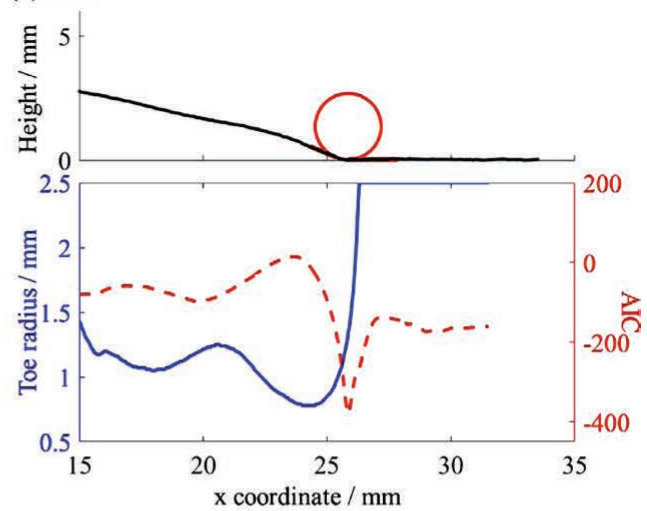

(d) CR2

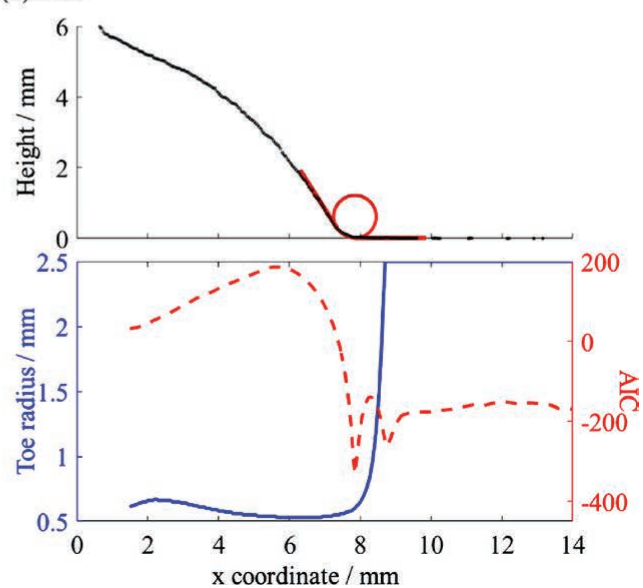

Fig. 2. Height profile in the direction perpendicular to the weld line, fitted toe radius and Akaike Information Criterion (AIC) in (a)-(b) butt joints and (c)-(d) cruciform joints. (Online version in color.) 
the lowest AIC value at each $x_{\mathrm{c}}$ position, respectively. It was clearly found that the AIC showed the lowest value at the toe position, and the circle representing the toe radius successfully matched the measurement data in all cases.

The calculation results were compared with the results of the conventional method. In the conventional method, a skilled technician estimated the geometrical parameters by manually fitting a circle to the same height profile data. The results are depicted in Fig. 3. Overall, both the toe radius and the reinforcement angle were in good agreement with the manual fitting. The toe radius determined by the AIC method showed a smaller value than the manual fitting by $0.09 \mathrm{~mm}$ to $0.2 \mathrm{~mm}$, whereas it has been reported that the toe radius measured by the different inspectors scattered with a standard deviation of approximately $0.2 \mathrm{~mm} .{ }^{17}$ ) Therefore, it can be concluded that the AIC method can estimate the toe radius with adequate accuracy. The average calculation time of the AIC method was $3.4 \mathrm{~s}$ for one profile with a single-core $2.3 \mathrm{GHz}$ processor. The advantage of the AIC method is that it can be performed automatically without professional skills, which will be useful for evaluating large numbers of height profiles. The following section presents the probability distribution of the geometrical parameters obtained by applying the proposed method to a number of height profiles in bead-on-plate welding. The limitation is that only two conventional shape parameters can be extracted in this simple geometrical model. As pointed out by Schork et al., ${ }^{16)}$ the fitting result of the toe radius strongly depends on the step width in the height profile because the shape of the weld toe can be fitted to circles at various scales. Since the shapes at various scales affect stress concentration and fatigue crack initiation life, a small number of conventional shape parameters are insufficient, and more statistical analysis of the weld toe geometry will be required to evaluate the fatigue properties of weld joint more generally.

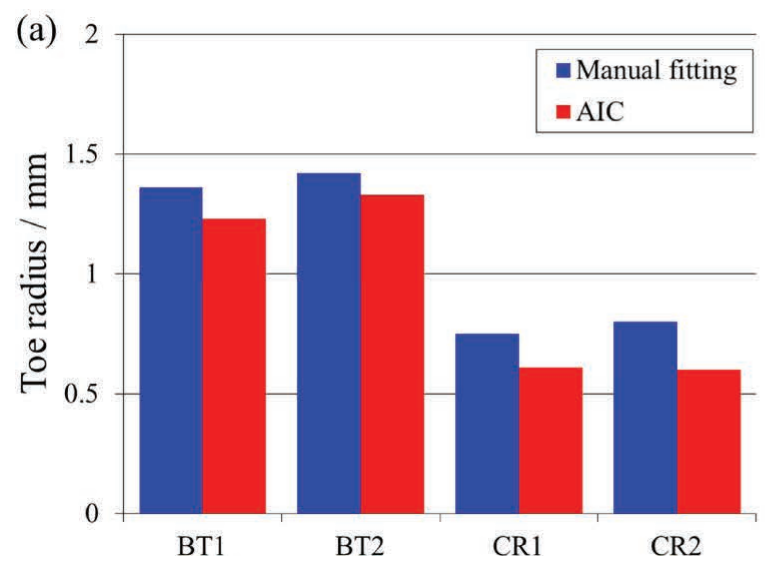

\section{Bayesian Optimization of Numerical Model for Welding}

\subsection{Bead-on-plate Welding}

In the following sections, a method for identifying heat source parameters in the welding simulation will be considered. To avoid misalignment problems in butt weld joints and cruciform joints, bead-on-plate welding was selected as an example. The bead-on-plate welding was conducted on a low carbon steel (JIS SM490YB) plate with MX-Z200 filler metal using a metal active gas (MAG) process. The welding voltage, current, and speed were $300 \mathrm{~V}, 35 \mathrm{~A}$ and $250 \mathrm{~mm} / \mathrm{min}$, respectively. The chemical compositions are listed in Table 1. A schematic of the configuration of bead-on-plate welding is shown in Fig. 4. The temperature history during the welding process was measured with four thermocouples at a position of approximately 5, 10, 15, 20 $\mathrm{mm}$ from the weld toe with a sampling period of $0.2 \mathrm{~s}$. The weld bead was deposited to both front and back surfaces at symmetrical positions in order to prevent a bending moment when conducting the fatigue test in future research. After welding, both ends of the plate including the beginning and end of the welding were cut out as shown in Fig. 4. The cross-sectional observation of the bead-on-plate specimen is shown in Fig. 5(a). Using a laser displacement meter,

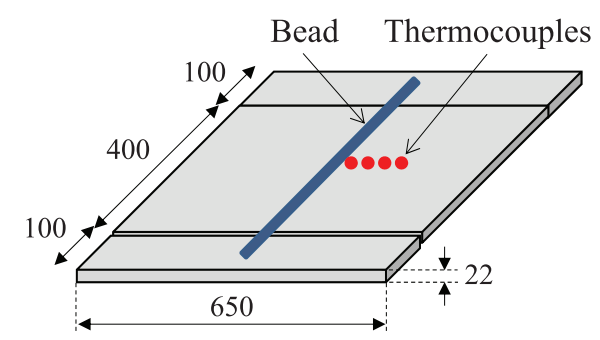

Fig. 4. Configuration of bead-on-plate welding. (Online version in color.)

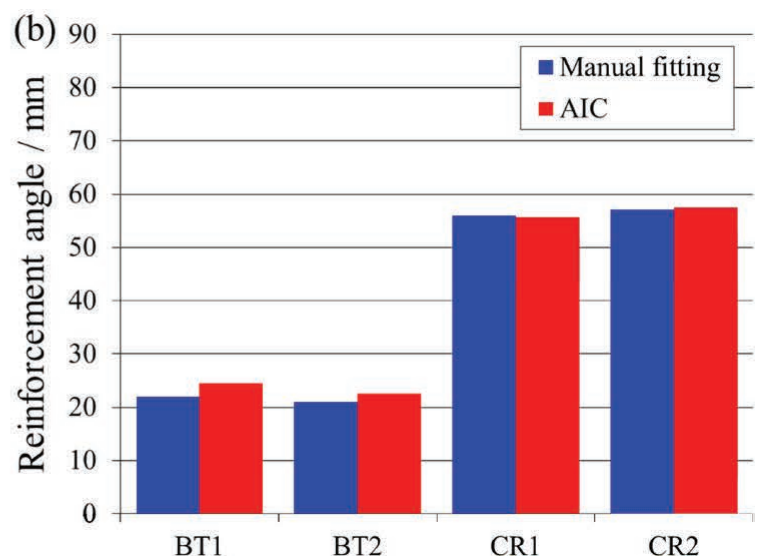

Fig. 3. Comparison of manual fitting and AIC method in the estimation of (a) toe radius and (b) reinforcement angle (BT: butt joint, CR: cruciform joint). (Online version in color.)

Table 1. Chemical compositions of base and filler metals (unit: mass\%).

\begin{tabular}{cccccccccc}
\hline Materials & $\mathrm{C}$ & $\mathrm{Si}$ & $\mathrm{Mn}$ & $\mathrm{P}$ & $\mathrm{S}$ & $\mathrm{A} 1$ & $\mathrm{~N}$ & $\mathrm{O}$ & $\mathrm{Nb}$ \\
\hline Base metal (JIS SM490B) & 0.164 & 0.27 & 1.40 & 0.018 & 0.0022 & 0.033 & 0.0030 & $<0.001$ & 0.026 \\
Filler metal (MX-Z200) & 0.083 & 0.44 & 1.53 & 0.013 & 0.0076 & 0.020 & 0.0036 & 0.070 & 0.011 \\
\hline
\end{tabular}


(a)

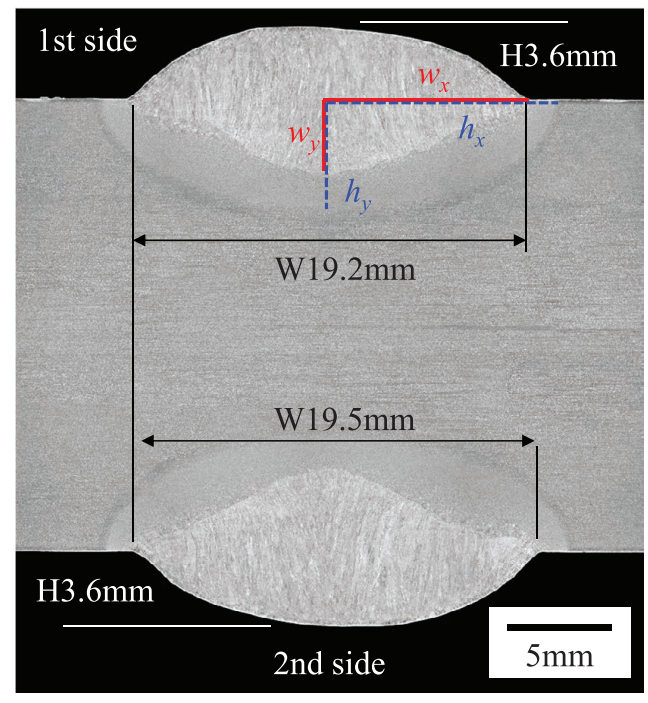

(b)

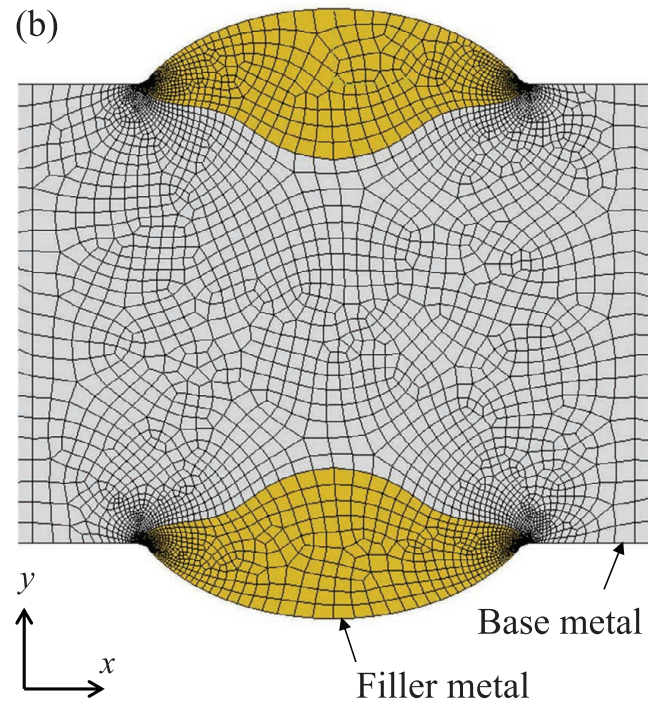

Fig. 5. (a) Cross sectional observation of bead-on-plate specimen and (b) finite element model for numerical simulation. The definitions of wx, wy, hx and hy in cross sectional observation are used in section 3.4. (Online version in color.)



Fig. 6. Histogram of toe radius of bead-on-plat weld extracted by AIC method and the fitted generalized extreme value (GEV) distribution. (Online version in color.)

50 height profiles along the perpendicular direction of the weld line were obtained with a step width of $0.05 \mathrm{~mm}$. The toe radius of each height profile was calculated by the AIC method proposed in the previous section. The probability distribution of the radius is plotted in Fig. 6. The solid line in the figure represents the fitted generalized extreme value (GEV) distribution.

\subsection{Finite Element Model}

A two-dimensional finite element model was created by inputting the global geometries (thickness and width of the plate) and the minimum experimental value of the toe radius obtained in the previous section and the corresponding reinforcement angle into the fatigue calculation framework ${ }^{14)}$ developed by the authors. The boundary lines between the filler metal and the base metal were manually defined by drawing Bezier curves. The created finite element model is shown in Fig. 5(b). The minimum mesh size at the weld toe was set to $0.01 \mathrm{~mm}$ which is approximately seven times smaller than the toe radius. The thermophysical and mechanical properties of the base metal and the filler metal were calculated from the chemical compositions using commercial software JMatPro v9.0. ${ }^{33)}$ The density, thermal conductivity, specific heat, Young's modulus, Poisson's ratio, yield stress, thermal expansion coefficient were obtained as a function of temperature for five phases of ferrite, pearlite, bainite, martensite, and austenite in the two materials. The heat transfer and the phase transformation were simultaneously computed by a strongly coupled method, and the temperature histories at four locations corresponding to the thermocouples in the experiment were recorded. The details of the calculation procedure can be found in reference. ${ }^{14)}$ In the heat transfer analysis, the distribution of the heat input was defined by the Goldak's double-ellipsoidal heat source model ${ }^{34)}$ where the front and rear heat input distributions are represented by three-dimensional Gaussian distributions as shown in Fig. 7. The heat input in the front and the rear of the heat source is expressed by the following equations as a function of coordinates and time:

$$
\begin{aligned}
& q(x, y, z, t)= \\
& \left\{\begin{array}{l}
\frac{6 \sqrt{3} f_{\mathrm{f}} Q}{a b c_{\mathrm{f}} \pi \sqrt{\pi}} \exp \left(-\frac{3 x^{2}}{a^{2}}\right) \exp \left(-\frac{3 y^{2}}{b^{2}}\right) \exp \left[-\frac{3\{z+v(\tau-t)\}^{2}}{c_{\mathrm{f}}^{2}}\right] \quad(z \leq v(\tau-t)) \\
\frac{6 \sqrt{3} f_{\mathrm{r}} Q}{a b c_{\mathrm{r}} \pi \sqrt{\pi}} \exp \left(-\frac{3 x^{2}}{a^{2}}\right) \exp \left(-\frac{3 y^{2}}{b^{2}}\right) \exp \left[-\frac{3\{z+v(\tau-t)\}^{2}}{c_{\mathrm{r}}^{2}}\right] \quad(z>v(\tau-t))
\end{array}\right.
\end{aligned}
$$

where $Q$ is energy input rate $(\mathrm{J} / \mathrm{s}), f_{\mathrm{f}}$ and $f_{\mathrm{r}}$ are fractions of the heat input in the front and rear quadrants $\left(f_{\mathrm{f}}+f_{\mathrm{r}}=\right.$ $2), v$ is welding speed $(\mathrm{m} / \mathrm{s}), \tau$ is a lag factor to define the initial position of the heat source, $a, b, c_{\mathrm{f}}$ and $c_{\mathrm{r}}$ are shape parameters shown in Fig. 7. The energy input rate $(Q)$ can be written as the product of the electrical power consumption and the conversion efficiency:

$$
Q=\eta V I,
$$

where $\eta$ is the conversion efficiency, $V$ is voltage, and $I$ is current value. Thus, it is necessary to properly determine six independent parameters $\left(a, b, c_{\mathrm{f}}, c_{\mathrm{r}}, f_{\mathrm{f}}\right.$ and $\left.\eta\right)$ in the welding simulation. To simplify the problem, it was assumed that the 


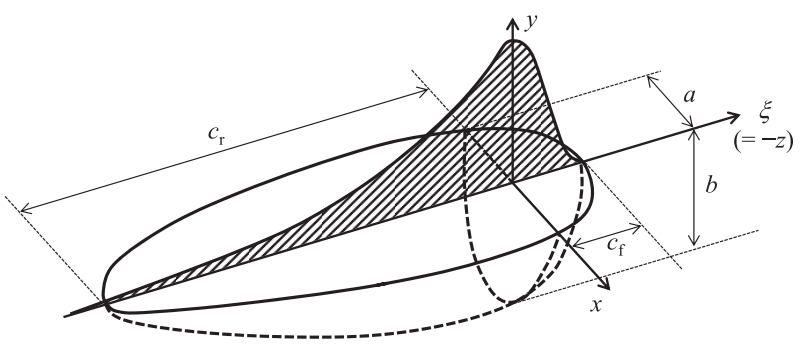

Fig. 7. Double ellipsoid heat source model for welding and definition of the shape parameters.

Table 2. Parameters in finite element simulation of welding.

\begin{tabular}{ccccccc}
\hline$V(\mathrm{~V})$ & $I(\mathrm{~A})$ & $v(\mathrm{~mm} / \mathrm{min})$ & $b$ & $c_{\mathrm{f}}$ & $c_{\mathrm{r}}$ & $f_{\mathrm{r}} / f_{\mathrm{f}}$ \\
\hline 300 & 35 & 250 & $a$ & $a$ & $2 a$ & 1.2 \\
\hline
\end{tabular}

shape parameters of $b, c_{\mathrm{f}}$, and $c_{\mathrm{r}}$ are proportional to $a$, and the fraction of heat inputs of $f_{\mathrm{f}}$ and $f_{\mathrm{r}}$ were determined by the widely used values of $f_{\mathrm{r}} / f_{\mathrm{f}}=1.2$. The parameters used in the heat transfer simulation were summarized in Table 2. Under these assumptions, there remain two independent parameters to be fitted, $a$ and $\eta$. In many cases, the fitting process is performed manually by trial and error, which limits the calculation efficiency and may lead to an inappropriate solution. To estimate optimal heat source parameters more efficiently, the application of Bayesian optimization will be considered in the next section.

\subsection{Bayesian Optimization}

3.3.1. Gaussian Process Regression (GPR) Model and Acquisition Function

Bayesian optimization is a general technique to solve inverse problems of finding input parameters that minimize (or maximize) the output of the objective function. Instead of directly optimizing the computationally expensive objective function, the stochastic regression model, and the acquisition function with low computational cost are utilized to find a promising solution of input parameters. The objective function in this study is the error in finite element simulations. The optimization is performed by repeating the following process:

(i) Predict the mean and standard deviation of the objective function by the Gaussian process regression (GPR) model.

(ii) Obtain the next search point by calculating the acquisition function using the mean and standard deviation of the GPR model.

(iii) Perform the calculation of the objective function at the obtained search point, and save the output of the objective function.

(iv) Update the GPR model.

These calculation procedures were repeated until reaching a certain number of iterations. The GPR provides a normal distribution (i.e. mean and standard deviation) of the objective function, unlike other regression methods. The acquisition function is a function that guides the search for minimizing the objective function. The efficiency of optimization generally depends on the choice of the kernel function of the GPR and acquisition function. The ARD Matérn 5/2 kernel, ${ }^{35)}$ which is widely used in the literature of Bayesian optimization, was used as the kernel function. Since there is no universal choice of the acquisition function, the following four types of acquisition functions were examined:

$$
\begin{gathered}
P I(\boldsymbol{\theta})=\max \left(\mathbf{y}_{\mathrm{obs}}\right)-\hat{y}(\boldsymbol{\theta}) \\
E I(\boldsymbol{\theta})=\left\{\max \left(\mathbf{y}_{\mathrm{obs}}\right)-\hat{y}(\boldsymbol{\theta})\right\} \Phi\left(\frac{\min \left(\mathbf{y}_{\mathrm{obs}}\right)-\hat{y}(\boldsymbol{\theta})}{\sigma(\boldsymbol{\theta})}\right) \\
+\sigma(\boldsymbol{\theta}) \phi\left(\frac{\min \left(\mathbf{y}_{\mathrm{obs}}\right)-\hat{y}(\boldsymbol{\theta})}{\sigma(\boldsymbol{\theta})}\right) \\
U C B(\boldsymbol{\theta})=\max \left(\mathbf{y}_{\mathrm{obs}}\right)-\hat{y}(\boldsymbol{\theta})+\kappa \sigma(\boldsymbol{\theta}) \ldots \ldots \ldots \ldots \\
M I_{t}(\boldsymbol{\theta})=\max \left(\mathbf{y}_{\mathrm{obs}}\right)-\hat{y}_{t}(\boldsymbol{\theta})+\kappa \\
\left(\sqrt{\sigma_{t}{ }^{2}(\boldsymbol{\theta})+\sum_{i=1}^{t-1} \sigma_{i}^{2}(\boldsymbol{\theta})}-\sqrt{\sum_{i=1}^{t-1} \sigma_{i}^{2}(\boldsymbol{\theta})}\right) \ldots
\end{gathered}
$$

where $\boldsymbol{\theta}$ is input variables, $\mathbf{y}_{\mathrm{obs}}$ is existing data of objective function, $\hat{y}(\boldsymbol{\theta})$ is mean value of objective function estimated by GPR model, $\phi$ is normal distribution, $\Phi$ is cumulative distribution function of the normal distribution, $\sigma$ is standard deviation estimated by GPR, $\kappa$ is constant and $t$ is the index of iteration number. Generally, in optimization procedures, there is a trade-off between "exploration" (acquisition of new knowledge) and "exploitation" (utilization of existing knowledge). Too much exploration can lead to an inefficient search, whereas the algorithm might be trapped in a local minimum with extreme exploitation. ${ }^{36)}$ In Bayesian optimization, the trade-off is controlled by the acquisition function. The probability of improvement (PI) is one of the most classical acquisition functions using only the mean value of the GPR model. The PI searches the point with the highest probability that the objective function is minimized. The next search point is represented by $\boldsymbol{\theta}^{(n+1)}=\arg \max \{P I(\boldsymbol{\theta})\}$. The expected improvement $(\mathrm{EI})^{37)}$ uses both mean and standard deviation obtained from the GPR model to conduct a search taking into account the trade-off between exploration and exploitation. The upper confidence bound (UCB) ${ }^{38)}$ directly uses the mean and standard deviation of the GPR model and controls the balance of exploration and exploitation by hyperparameter of $\kappa$. In the current analysis, $\kappa$ was set to 2. The mutual information $(\mathrm{MI})^{39)}$ is a relatively recently proposed method that uses a history of mean and standard deviation and has less influence of hyperparameter $\kappa$ than UCB. The literature value of $\kappa=2.51^{39)}$ was used in the MI function.

\subsubsection{Estimation of Heat Source Parameter Using Tem- perature History}

The optimization problem of the heat source variables, $\boldsymbol{\theta}=(a, \eta)$, to minimize the difference in temperature history between the experiment and simulation can be formulated using the objective function, $E_{\text {temp }}(\theta)$, as follows:

$$
\begin{aligned}
E_{\mathrm{temp}}(\theta) & =\sqrt{\sum_{i}\left(T_{\mathrm{sim}, i}-T_{\mathrm{exp}, i}\right)^{2}} . \\
\hat{\boldsymbol{\theta}} & =\underset{\theta \in \Theta}{\arg \min } E(\boldsymbol{\theta}) \ldots \ldots \ldots \ldots \ldots
\end{aligned}
$$


where the objective function, $E_{\text {temp }}(\boldsymbol{\theta})$, indicates root mean squared error (RMSE) of temperature from the beginning to the end of welding at four positions, $i$ is data index, $T_{\mathrm{sim}, i}$ is temperature calculated by FEM, $T_{\text {exp }, i}$ is temperature measured by thermocouples, $\Theta$ is a searching range of input variables and $\hat{\boldsymbol{\theta}}$ is the heat source variables to minimize the RMSE. The searching range was set to $0.1 \leq a \leq 30 \mathrm{~mm}$ and $0 \leq \eta \leq 1$ from consideration of the fusion zone size shown in Fig. 5(a). To reduce the calculation time, the time increment size in FEM was not fixed and was automatically determined by Sysweld software. ${ }^{40)}$ Since the experimental values of temperature were measured at a constant sampling rate, linear interpolation was used to estimate the experimental temperature at the time corresponding to the calculation point in FEM, and the RMSE was calculated according to Eq. (11). The Bayesian optimization was performed using the four types of acquisition functions described above, and the results were compared.

3.3.3. Estimation of Heat Source Parameter Using Both Temperature History and Shape of Bead and Heataffected Zone

To fill the lack of temperature information inside the plate, another objective function, $E(\boldsymbol{\theta})$, was defined by considering the shape of the fusion zone and heat-affected zone (HAZ) as follows:

$$
\begin{aligned}
& E_{\mathrm{geom}}= \\
& \sqrt{\left(w_{\mathrm{x}, \mathrm{FEM}}-w_{\mathrm{x}, \mathrm{exp}}\right)^{2}+\left(w_{\mathrm{y}, \mathrm{FEM}}-w_{\mathrm{y}, \mathrm{exp}}\right)^{2}+\left(h_{\mathrm{x}, \mathrm{FEM}}-h_{\mathrm{x}, \exp }\right)^{2}+\left(h_{\mathrm{y}, \mathrm{FEM}}-h_{\mathrm{y}, \mathrm{exp}}\right)^{2}}
\end{aligned}
$$

$$
E(\boldsymbol{\theta})=\frac{E_{\text {temp }}(\theta)}{\delta_{\text {temp }}}+\frac{E_{\text {geom }}(\theta)}{\delta_{\text {geom }}} .
$$

where $w_{\mathrm{x}}$ and $w_{\mathrm{y}}$ are $\mathrm{x}$ and $\mathrm{y}$ coordinates of fusion line, $h_{\mathrm{x}}$ and $h_{\mathrm{y}}$ are $\mathrm{x}$ and $\mathrm{y}$ coordinates of $\mathrm{Ac}_{3}$ line as shown in Fig. $5(\mathrm{a}), E_{\text {temp }}(\boldsymbol{\theta})$ is defined by Eq. (11), $\delta_{\text {temp }}$ is measurement accuracy of temperature, and $\delta_{\text {geom }}$ is measurement accuracy of the geometry of fusion and $\mathrm{Ac}_{3}$ lines. The accuracies were defined as $\delta_{\text {temp }}=10^{\circ} \mathrm{C}$ and $\delta_{\text {geom }}=0.2 \mathrm{~mm}$. The EI function was utilized as the acquisition function in the optimization to avoid falling into local minima.

\subsection{Results and Discussion}

In order to confirm the features of the four acquisition functions, simple one-dimensional Bayesian optimization was conducted by fixing the heat source size $(a)$ to $10 \mathrm{~mm}$ and changing only the efficiency $(\eta)$. The objective function of $E_{\text {temp }}(\theta)$ and the acquisition function after four FEM calculations are plotted in Fig. 8. The open circle in the upper plot represents the RMSE calculated by FEM, and the black solid line and dashed lines are mean and $95 \%$ confidence interval (CI) of the GPR model, respectively. The acquisition functions calculated from the GPR model are plotted on the lower side. As is apparent from Eq. (7), the PI value is maximized when the mean value of the GPR model is minimized. As a result, the PI tends to overexploit in many situations. ${ }^{41)}$ The other acquisition functions of EI, UCB, and MI show more complicated behavior by considering the standard deviation. For example, MI selected the next search point in the vicinity of $x=0.8$ which is still not searched.

Two-dimensional Bayesian optimization was then per-

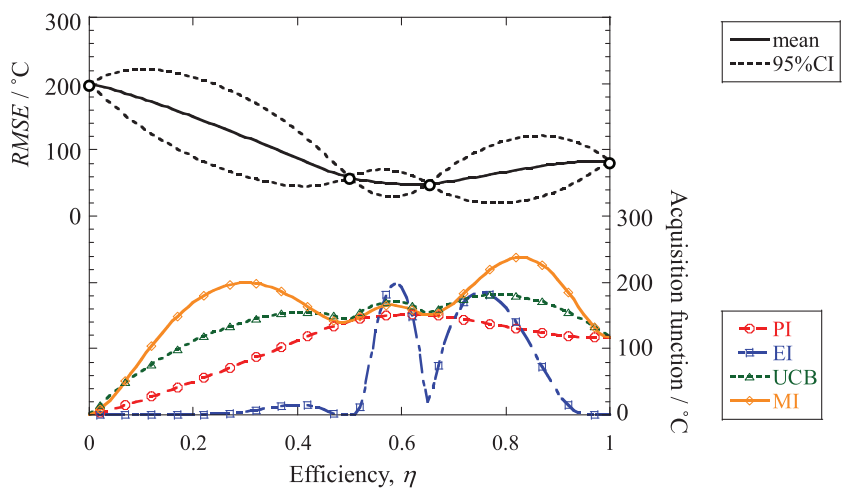

Fig. 8. Example of one-dimensional Bayesian optimization with Gaussian process regression using different acquisition functions (PI: probability of improvement, EI: expected improvement, UCB: upper confidence bound, MI: mutual information). The heat source size was fixed to $10 \mathrm{~mm}$. (Online version in color.)

formed using only the temperature history by changing both heat source size and conversion efficiency. As the initial values, the RMSE was calculated in advance at nine points of $(a(\mathrm{~mm}), \eta)=(10,0),(20,0),(30,0),(10,0.5)$, $(20,0.5),(30,0.5),(10,1.0),(20,1.0)$ and $(30,1.0)$. All procedures of optimization including finite element simulation, temperature history extraction, calculations of RMSE, GPR and acquisition functions are performed automatically by Python scripts. The series of calculation procedures took approximately one minute for one loop with a singlecore $2.3 \mathrm{GHz}$ processor. The number of iterations was set to 100 in consideration of the total calculation time. The acquisition functions and RMSE after the 100 iterations were depicted as a function of $a$ and $\eta$ in Figs. 9 and 10, respectively. Overall, the search started in the vicinity of the center of the initial points and approached the final solution in all acquisition functions. The final solution is depicted as open white circle in the figure. In the case of PI, the search point simply moved in the direction of decreasing the RMSE because the PI considers only exploitation. The EI and UCB functions provided more aggressive searches than the PI function by considering the standard deviation of the GPR model. The MI function first evaluated the extreme conditions such as $(a(\mathrm{~mm}), \eta)=(0.1,1.0)$ and $(0.1,0.655)$, and then showed monotonic behavior similar to the PI function. This is because the MI is designed to conduct exploration in the beginning and exploitation in a later stage by using the standard deviation history in the iterations. In all acquisition functions, the combination of $(a(\mathrm{~mm}), \eta)=(2.2,0.805)$ was finally selected to minimize the RSME of temperature. In the current calculation, the heat source size was much smaller than the size of the fusion zone (radius of the fusion zone was $9.6 \mathrm{~mm}$ ). The calculation efficiency of each acquisition function was evaluated by cumulative regret ${ }^{38)}$ which is defined by the following equation:

$$
R_{t}=\sum_{t}\left\{y\left(\theta_{t}\right)-y\left(\boldsymbol{\theta}^{*}\right)\right\}
$$

where $y\left(\boldsymbol{\theta}_{t}\right)$ is the objective function at iteration number of $t, y\left(\theta^{*}\right)$ is the true minimum value of the objective function. The $y\left(\boldsymbol{\theta}_{t}\right)-y\left(\boldsymbol{\theta}^{*}\right)$ is referred to as "regret." The true minimum value, $y\left(\boldsymbol{\theta}^{*}\right)$, was calculated by the finally selected 
(a)



(c)



(b)

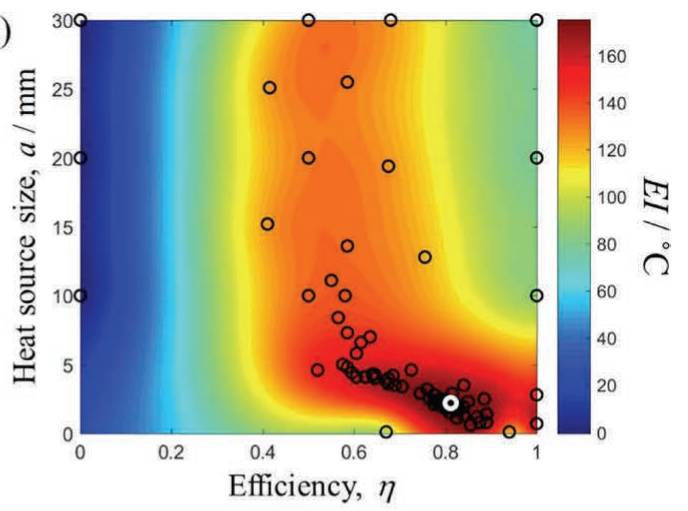

(d)

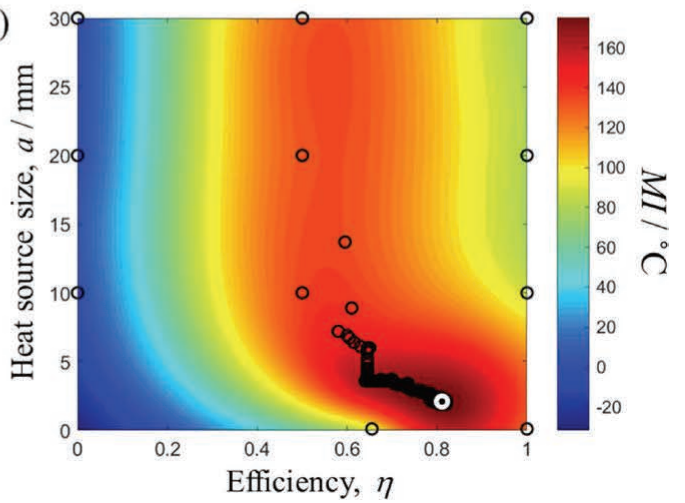

Fig. 9. Distribution of acquisition function after 100 iterations: (a) probability of improvement, (b) expected improvement, (c) upper confidence bound, and (d) mutual information. Open black circles represent points evaluated by finite element analysis, and open white circle represents the final solution. (Online version in color.)

(a)

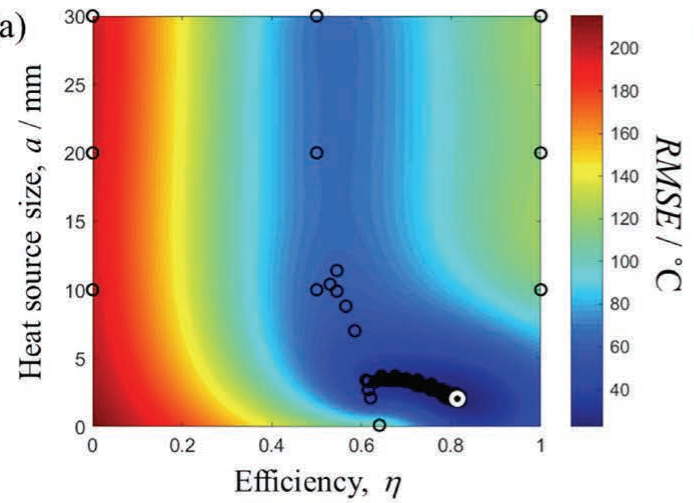

(c)

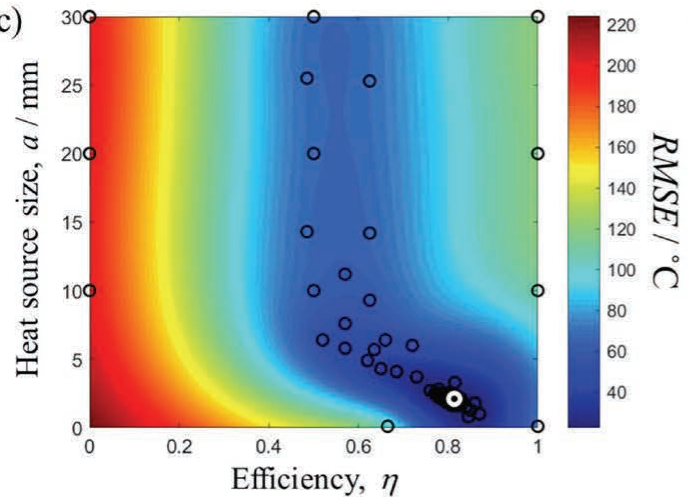

(b)

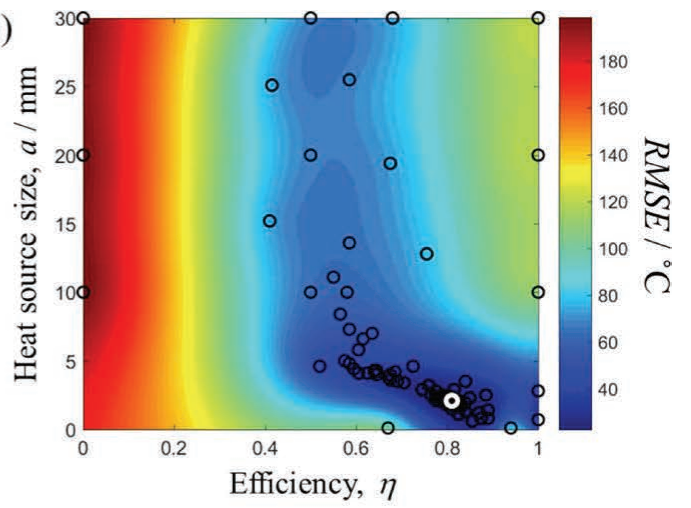

(d)

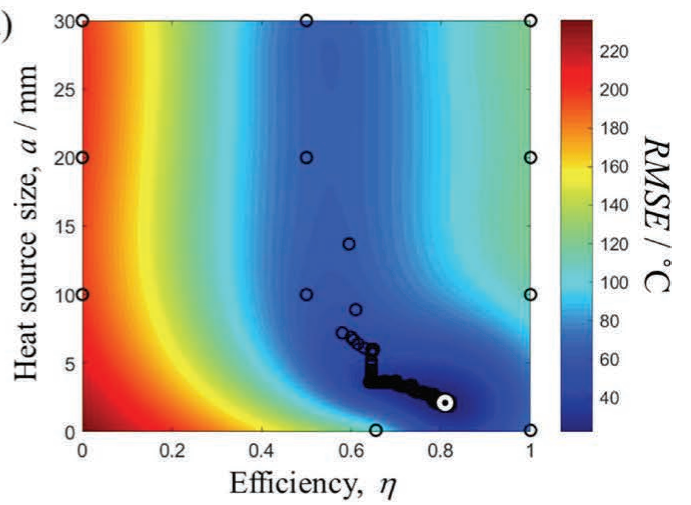

Fig. 10. Distribution of root mean squared error (RMSE) after 100 iterations: (a) probability of improvement, (b) expected improvement, (c) upper confidence bound, and (d) mutual information. Open black circles represent points evaluated by finite element analysis, and open white circle represents the final solution. (Online version in color.)

input values of $(a(\mathrm{~mm}), \eta)=(2.2,0.805)$. The cumulative regrets are plotted against the iteration number in Fig. 11. The closed circles on the cumulative regret curves represent the number of iterations when the true minimum value was obtained. The smaller accumulated regret suggests a more efficient search in the optimization. In the figure, the PI and 
UCB showed better performances than the other acquisition functions. This result is in contrast to the previous study where the EI and MI show lower regret. ${ }^{39)}$ This is due to the difference in the nature of the objective function. In the current optimization problem, it seems that the objective function has only one local minimum as shown in Fig. 10, whereas more complicated objective functions with multiple local minima were examined in the reference. ${ }^{39)}$

The temperature history calculated at the optimized condition is plotted with the experimental data in Fig. 12(a). The calculated results are in good agreement with the experimental data. To evaluate the temperature distribution in more detail, the fusion line and $\mathrm{Ac}_{3}$ line were extracted from the experimental observation (Fig. 5(a)), and compared with the calculation result. The results are shown in Fig. 12(b). The fusion line and $\mathrm{Ac}_{3}$ line in the calculation were defined as the lines where the maximum temperature corresponds to the melting point $\left(1474^{\circ} \mathrm{C}\right)$ and $\mathrm{Ac}_{3}$ temperature $\left(812^{\circ} \mathrm{C}\right)$, respectively. The melting point and $\mathrm{Ac}_{3}$ temperature were calculated by JMatPro. The discrepancy between the experiment and calculation in the two lines increased with the distance from the surface. This is because the experimental data of the temperature history is given only on the surface in the optimization.

The results of optimization using both temperature and fusion and $\mathrm{Ac}_{3}$ lines are shown in Fig. 13. The solution was

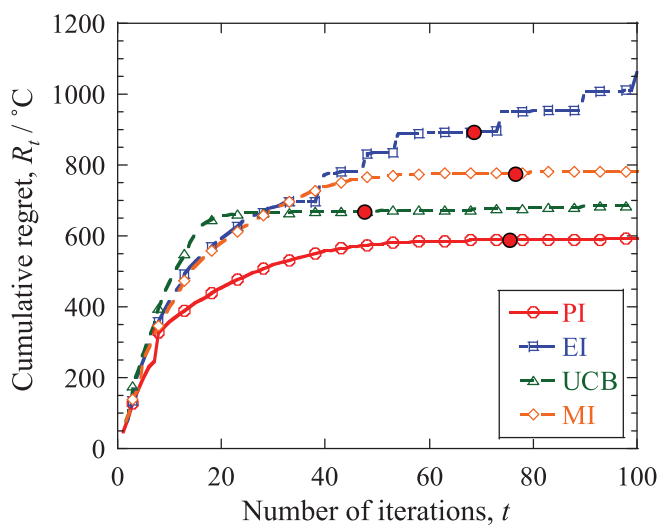

Fig. 11. Cumulative regret in Bayesian optimization using temperature history. Closed circles represent number of iterations when the minimum solution was obtained. (Online version in color.) determined to be $(a, \eta)=(6.9,0.655)$. The heat source size became a reasonable value, and the conversion efficiency was relatively small compared with the literature value. In the literature, the conversion efficiency for MAG welding was defined between 0.6 and $0.85 .{ }^{42-44)}$ The temperature history and fusion and $\mathrm{Ac}_{3}$ lines are plotted in Fig. 14. Compared with the results in the previous section (Fig. 12), although the error in temperature history became slightly larger, the accuracy of the shape of the fusion zone and HAZ is considerably improved. Focusing on the temperature history, the measured temperature histories were much lower than the $\mathrm{Ac}_{3}$ temperature and melting point because of the positions of thermocouples. If the experimental data of the temperature history inside the bead or in the immediate vicinity of the bead are obtained, it may provide the optimal combination of $(\alpha, \eta)$ without shapes of weld bead and HAZ. On the other hand, measuring the temperature in the vicinity of bead is not always easy. The method proposed in this study is useful in that the heat source parameters can be estimated from temperature history at a position away from the bead and weld bead shape, which can be easily acquired experimentally.

From the above results, it was demonstrated that the heat source parameters could be automatically estimated by

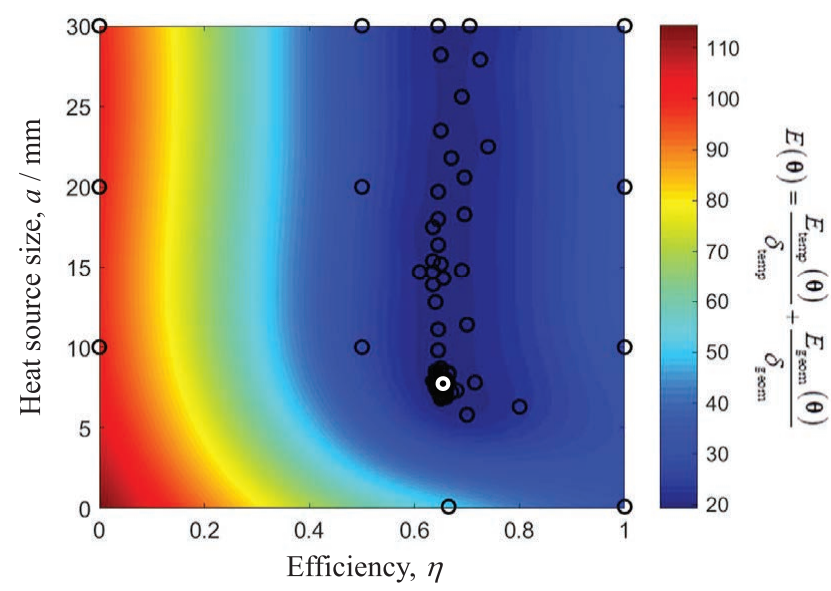

Fig. 13. Distribution of root mean squared error (RMSE) considering temperature history and shape of bead and heat-affected zone. Open black circles represent points evaluated by finite element analysis, and open white circle represents the final solution. (Online version in color.) (a)



(b)

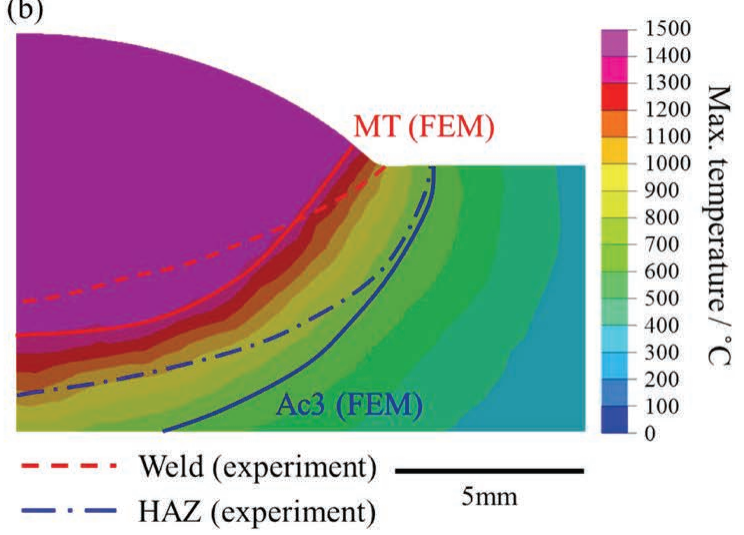

Fig. 12. Simulation results by minimizing errors in temperature history: (a) Temperature history and (b) maximum temperature distribution, fusion line and Ac3 line. (Online version in color.) 
(a)

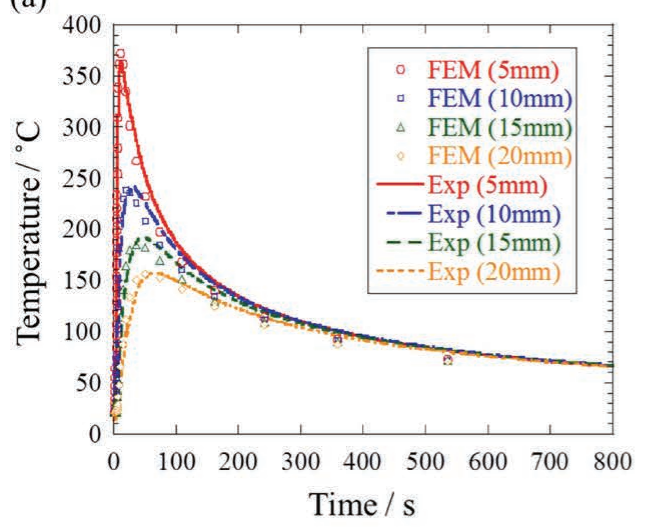

(b)

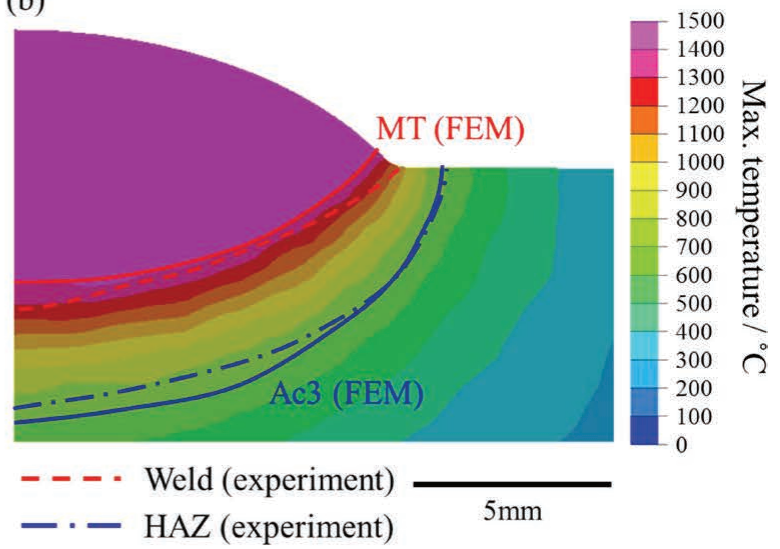

Fig. 14. Simulation results by minimizing errors in temperature history and shape of weld bead and heat affected zone (HAZ): (a) Temperature history and (b) maximum temperature distribution, fusion line and Ac3 line. (Online version in color.)
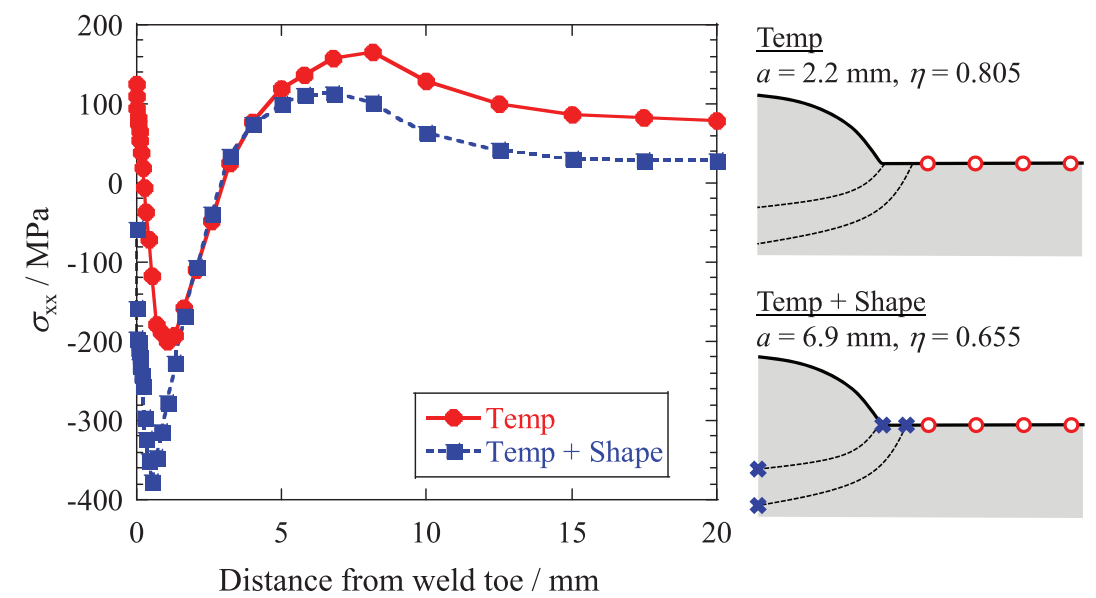

$a=6.9 \mathrm{~mm}, \eta=0.655$



Distance from weld toe / $\mathrm{mm}$

Fig. 15. Residual stress components perpendicular to the weld line after the first pass welding calculated with two optimized models. (Online version in color.)

Bayesian optimization with the objective function of $E(\boldsymbol{\theta})$. In the current analysis, the heat source model was simplified to two parameters by assuming the similarity of the heat source shape because the number of calculations required for the optimization increases exponentially with the dimension of the input variables. Recently, Bayesian optimization methods for solving high-dimensional problems have been intensively studied, and it has been shown that the dropout strategy used in neural networks is effective also in Bayesian optimization. ${ }^{45)}$ Using the high-dimensional Bayesian optimization, the better heat source model would be obtained by adding the front and rear shape parameters and the energy ratio into the input variables.

Different values of heat source parameters were obtained between the optimization methods considering only the surface temperature history (Fig. 10) and both the temperature history and the shape of the fusion zone and HAZ (Fig. 13). The calculated temperature history on the surface was similar for both heat source parameters, whereas the results of the fusion and $\mathrm{Ac}_{3}$ lines are quite different. These results raise a question about the effect of heat source parameters on residual stress calculation. Using the two combinations of heat source parameters, the residual stress was calculated. Similar to the experiment, the boundary conditions that both ends of the plate were rigidly constrained during the welding were assigned, and the stress distribution after releasing the constraint was regarded as the residual stress. The residual stress components perpendicular to the weld line calculated with two optimized models are plotted in Fig. 15. Although the absolute values of residual stress were different between the two models, the tendencies of distributions were similar in the two models and consistent with the results of neutron diffraction measurement. ${ }^{46)}$ In order to evaluate these results in more detail, it is necessary to measure residual stress distribution of the welded joint used in the current study by experimental methods such as mechanical cutting (stress release method), X-ray diffraction and neutron diffraction.

\section{Conclusions}

Data assimilation techniques for weld toe geometry and heat source model during welding were proposed and applied to experimental data. The following conclusions can be drawn.

(1) The toe radius and the reinforcement angle were automatically extracted from the surface height profile of the butt weld joint and cruciform joint using Akaike's information criterion (AIC). The extracted values showed good agreement with the manual fitting.

(2) The heat source parameters in welding simulation 
were determined by Bayesian optimization. The cumulative regret was compared for four types of acquisition functions (PI, EI, UCB, and MI). Due to the simplicity of the optimization problem, the PI and UCB showed better performances than the other acquisition functions.

(3) The objective function using only the temperature history provided a relatively small size of the heat source. The temperature history on the surface was consistent with the experimental data whereas the fusion and $\mathrm{Ac}_{3}$ lines did not fit the experimental results.

(4) By including both the temperature and the shape of the fusion zone and HAZ in the objective function, reasonable heat source size was obtained. Both temperature history and fusion and $\mathrm{Ac}_{3}$ lines were roughly fitted to the experimental results. Also, the profile of residual stress distribution was consistent with experimental results measured by the neutron diffraction method.

(5) It was demonstrated that a numerical model for welding simulation could be created with less manual works by using the above two data assimilation techniques.

\section{Acknowledgments}

This work was partially supported by Council for Science, Technology and Innovation (CSTI), Cross-ministerial Strategic Innovation Promotion Program (SIP), Structural Materials for Innovation (Funding agency: JST) and ISIJ Research Promotion Grant.

\section{REFERENCES}

1) I. Poutiainen and G. Marquis: Int. J. Fatigue, 28 (2006), 1037. https:// doi.org/10.1016/j.ijfatigue.2005.11.007

2) D. Radaj, C. M. Sonsino and W. Fricke: Fatigue Assessment of Welded Joints by Local Approaches, Woodhead Publishing, Cambridge, UK, (2006), 107.

3) J. L. Otegui, H. W. Kerr, D. J. Burns and U. H. Mohaupt: Int. J. Press. Vessel. Pip., 38 (1989), 385. https://doi.org/10.1016/0308-0161 (89)90048-3

4) A. Ohta, N. Suzuki, Y. Maeda, K. Hiraoka and T. Nakamura: Int. J. Fatigue, 21 (1999), 5113. https://doi.org/10.1016/S01421123(99)00062-6

5) J. A. Wasynczuk, R. O. Ritchie and G. Thomas: Mater. Sci. Eng., 62 (1984), 79. https://doi.org/10.1016/0025-5416(84)90269-6

6) H.-a. Nishikawa and Y. Furuya: Tetsu-to-Hagané, 105 (2019), 105 https://doi.org/10.2355/tetsutohagane.TETSU-2018-096

7) M. Madia, U. Zerbst, H. Th. Beier and B. Schork: Eng. Fract. Mech., 198 (2018), 171. https://doi.org/10.1016/j.engfracmech.2017.08.033

8) U. Zerbst, M. Madia, M. Vormwald and H. T. Beier: Eng. Fract. Mech., 198 (2018), 2. https://doi.org/10.1016/j.engfracmech.2017.04.030

9) K. Shibanuma, K. Ueda, H. Ito, Y. Nemoto, M. Kinefuchi, K Suzuki and M. Enoki: Mater. Des., 139 (2018), 269. https://doi. org/10.1016/j.matdes.2017.10.069

10) H. Ito, Y. Suzuki, H. Nishikawa, M. Kinefuchi, M. Enoki and K. Shibanuma: Int. J. Mech. Sci., 170 (2020), 105339. https://doi. org/10.1016/j.ijmecsci.2019.105339

11) F. Briffod, T. Shiraiwa and M. Enoki: Mater. Trans., 57 (2016), 1741 https://doi.org/10.2320/matertrans.M2016216

12) F. Briffod, T. Shiraiwa and M. Enoki: Mater. Sci. Eng. A, 695 (2017), 165. https://doi.org/10.1016/j.msea.2017.04.030

13) F. Briffod, T. Shiraiwa and M. Enoki: Int. J. Fatigue, 107 (2018), 72 https://doi.org/10.1016/j.ijfatigue.2017.10.019

14) T. Shiraiwa, F. Briffod and M. Enoki: Eng. Fract. Mech., 198 (2018), 158. https://doi.org/10.1016/j.engfracmech.2017.11.012

15) C. Beckmann, T. Kennerknecht, J. Preußner, M. Farajian, M. Luke and J. Hohe: Eng. Fract. Mech., 198 (2018), 142. https://doi. org/10.1016/j.engfracmech.2017.08.008

16) B Schork, P. Kucharczyk, M. Madia, U. Zerbst, J. Hensel, J. Bernhard, D. Tchuindjang, M. Kaffenberger and M. Oechsner: Eng. Fract. Mech., 198 (2018), 103. https://doi.org/10.1016/j. engfracmech.2017.07.001

17) K. Ishikawa, S. Fujimoto and K. Tateishi: Proc. 63rd Annual Conf. of the Japan Society of Civil Engineers, Japan Society of Civil Engineers, Tokyo, (2008), 973 (in Japanese).

18) M. Islam, A. Buijk, M. Rais-Rohani and K. Motoyama: Finite Elem. Anal. Des., 84 (2014), 54. https://doi.org/10.1016/j.finel.2014.02.003

19) G. Evensen: Data Assimilation: The Ensemble Kalman Filter, Springer Science \& Business Media, New York, (2009), 12.

20) J. i. Fukuda, S. i. Miyazaki, T. Higuchi and T. Kato: Geophys. J. Int., 173 (2008), 25. https://doi.org/10.1111/j.1365-246X.2007.03722.x

21) H. Nagao and T. Higuchi: Proc. 16th Int. Conf. on Information Fusion, Institute of Electrical and Electronics Engineers (IEEE), Piscataway, NJ, (2013), 1372.

22) Y. Wang, K. Satake, T. Maeda and A. R. Gusman: Earth Planets Space, 70 (2018), 131. https://doi.org/10.1186/s40623-018-0905-6

23) Y. Oka, M. Ohno and K. Matsuura: Tetsu-to-Hagané, 103 (2017), 755 (in Japanese). https://doi.org/10.2355/tetsutohagane.TETSU-2017-031

24) A. Solomou, G. Zhao, S. Boluki, J. K. Joy, X. Qian, I. Karaman, R. Arróyave and D. C. Lagoudas: Mater. Des., 160 (2018), 810. https:// doi.org/10.1016/j.matdes.2018.10.014

25) P. Fernandez-Zelaia, V. Roshan Joseph, S. R. Kalidindi and S. N. Melkote: Mater. Des., 147 (2018), 92. https://doi.org/10.1016/j. matdes.2018.03.037

26) K. Sasaki, A. Yamanaka, S.-i. Ito and H. Nagao: Comput. Mater. Sci., 141 (2018), 141. https://doi.org/10.1016/j.commatsci.2017.09.025

27) A. Yamanaka, Y. Maeda and K. Sasaki: Mater. Des., 165 (2019), 107577. https://doi.org/10.1016/j.matdes.2018.107577

28) S.-i. Ito, H. Nagao, T. Kurokawa, T. Kasuya and J. Inoue: Phys. Rev. Mater., 3 (2019), 053404. https://doi.org/10.1103/ PhysRevMaterials.3.053404

29) H. Akaike: 2nd Int. Symp. on Information Theory, Akademiai Kiado, Budapest, (1973), 267.

30) H. Akaike: IEEE Trans. Autom. Control, 19 (1974), 716

31) N. Maeda: Zisin (J. Seismol. Soc. Jpn. 2nd Ser.), 38 (1985), 365 (in Japanese). https://doi.org/10.4294/zisin1948.38.3 365

32) P. Sedlak, Y. Hirose, S. A. Khan, M. Enoki and J. Sikula: Ultrasonics, 49 (2009), 254. https://doi.org/10.1016/j.ultras.2008.09.005

33) Sente Software Ltd.: JMatPro User's Guide 9.0, Sente Software Ltd., Guildford, UK, (2016), 1.

34) J. Goldak, A. Chakravarti and M. Bibby: Metall. Trans. B, 15 (1984), 299. https://doi.org/10.1007/BF02667333

35) J. Snoek, H. Larochelle and R. P. Adams: Advances in Neural Information Processing Systems 25 (NIPS 2012), Curran Associates Inc., New York, (2012), 2951

36) E. Brochu, V. M. Cora and N. De Freitas: arXiv: 1012.2599, (2010), https://arxiv.org/ars/1012.2599, (accessed 2020-02-08).

37 D. R. Jones, M. Schonlau and W. J. Welch: J. Global Optim., 13 (1998), 455. https://doi.org/10.1023/A:1008306431147

38) N. Srinivas, A. Krause, S. Kakade and M. Seeger: Proc. 27th Int. Conf. on Machine Learning, Omnipress, Bellevue, WA, (2010), 1015.

39) E. Contal, V. Perchet and N. Vayatis: 31st Int. Conf. on Machine Learning, Institute of Electrical and Electronics Engineers (IEEE), New York, NY, (2014), 253.

40) ESI Group: Sysweld 2016 Reference Manual, ESI Group, Paris, (2016), 1.

41) J. Berk, V. Nguyen, S. Gupta, S. Rana and S. Venkatesh: Machine Learning and Knowledge Discovery in Databases, Springer International Publishing, Cham, (2019), 621.

42) T. Yamamoto, Y. Tsuji, F. Miyasaka and T. Ohji: Q. J. Jpn. Weld. Soc., 19 (2001), 628. https://doi.org/10.2207/qijws.19.628

$43)$ N. Chriestensen, V. Davies and K. Gjermundsen: Br. Weld. J., 12 (1965), 54

44) S. Okano and M. Mochizuki: ISIJ Int., 57 (2017), 511. https://doi. org/10.2355/isijinternational.ISIJINT-2016-394

45) C. Li, S. Gupta, S. Rana, T. V. Nguyen, S. Venkatesh and A. Shilton: IJCAI 2017: Proc. 26th Int. Joint Conf. on Artificial Intelligence, Association for the Advancement of Artificial Intelligence, Menlo Park, CA, (2017), 2096.

46) J. A. Francis, M. Turski and P. J. Withers: Mater. Sci. Technol., 25 (2009), 325. 